\title{
Broadband access to the Internet - an overview
}

\author{
H. Leopold \\ Alcatel \\ Scheydgasse 41, A-1211 Vienna, Austria, \\ Tel.: +43127722 3551, Fax.: +43127722 1172, \\ E-mail: helmut.leopold@aut.alcatel.at
}

\begin{abstract}
The evolution of the modern telecommunication environment depends on three main factors: (i) the market demand for new services, (ii) the politically stimulated liberalisation of the telecommunication market, and (iii) the technological advances of new telecommunication technologies. The technological advances include getting the fibre closer to the subscribers, cheaper equipment costs and standardisation.

Network operators are forced nowadays to enable, by offering new services, very high profit in a very short period of time with limited investment. For this reason, especially the access network and the technologies linked to it have an increasing importance for the economic success of an operator in a liberalised telecommunication market.

This article describes the most distinguishing aspects of an access network, its positioning within a telecommunication system, and the most important technological developments in this field: Fibre In The Loop (FITL) systems, Digital Subscriber Line (xDSL) technologies on copper twisted pair and the Hybrid Fibre Coax (HFC) technology on CATV networks.
\end{abstract}

Keywords

Access network, fibre in the loop (FITL), xDSL, ADSL, HFC, CATV, cable telephony, cable data modem

The original version of this chapter was revised: The copyright line was incorrect. This has been corrected. The Erratum to this chapter is available at DOI: $10.1007 / 978-0-387-35388-3 \_42$ 


\section{INTRODUCTION}

The evolution of the modern telecommunication environment depends on three main factors:

- the technological advances of new telecommunication technologies,

- the market demand for new services, and

- the politically stimulated liberalisation of the telecom market.

Especially the liberalisation in the telecommunications market has a tremendous impact on business in this area. New actors like international network operators, Cable TV (CATV) network operators, energy suppliers, railway organisations, city communication operators, etc. will start to offer telecommunication services in competition to the incumbent national network operator. This will have an impact on the market share, the tariff structure, the Quality of Service (QoS) and the offered services to the customers itself. The final target is the so called "Full Service Network (FSN)", which is capable of offering all types of interactive multimedia services at any time, any place and any QoS.

The provisioning of new telecommunication services in general and new multimedia services in particular is made possible by the availability of several new technologies as well as advances in standardisation:

- Optical signal transmission and SDH technology which allows the establishment of powerful backbone networks.

- New switching and transmission technologies like ATM which allows the transport of information, independent of their nature, and which allows dynamic establishment of high-bandwidth connections with flexible Quality of Service (QoS), in order to meet the application requirements. However, the recently initiated discussion concerning the integration of Internet- and ATMtechnologies will have an enormous impact on future developments in this field (Sales, 1998).

- Low cost digital mass storage permits economical deployment of digital video servers.

- Powerful video compression techniques like MPEG effectively reduce storage expense and transmission bandwidth demand.

- New wireless technologies for access and transmission networks based on satellite or terrestrial.

- New access network technologies to bring multimedia capacity over the last mile to a broad range of subscribers.

These technological advances are to a large extent also stimulated by the regulatory changes around the world for more open competition, liberalisation, and privatisation. Operators world wide are faced with this changing environment and are now in process to upgrade their network infrastructures in order to become a competitive network operator in the new telecommunication environment.

The question is now, how all these providers will transform their network systems and their organisations. Network operators are forced to maximise the revenue in a very short period of time with a limited investment. For this reason, 
especially the access network technologies linked to it have an increasing importance for the economic success of an operator in the liberalised telecom market. The access network represents an essential part of the overall costs of a telecommunication infrastructure. The investment cost are up to $70 \%$ for the access network and the ownership of the access network, i.e. the Operation and Maintenance (OAM) is up to $80 \%$ of the total cost. OAM costs include preventive and corrective maintenance, network management and repair of defective equipment.

In the past years there has been an international effort from telecommunication operators and manufacturers to maximise consensus on the systems required in the local access network to deliver a full set of telecommunications services, both narrowband and broadband (FSAN, 1997).

This article discusses the positioning of an access network in an overall telecommunication system and presents the recent advances of access technologies, where beside Fibre In The Loop (FITL) systems especially Digital Subscriber Line (DSL) technologies for twisted-pair telephone lines and Hybrid Fibre Coax (HFC) technology for coaxial CATV networks which allow the utilisation of existing access network infrastructures in order to offer broadband services additionally, are of main importance.

\section{EVOLUTION OF THE ACCESS NETWORK ARCHITECTURE}

The general trend in telecommunication networks is to establish powerful backbone networks which are composed of only a few network nodes. These nodes are interconnected by broadband connections and have an extensive access network, reducing the public switching hierarchy levels (Berkowitz, 1997).

All network operators which are running fibre optic networks, have established such powerful backbone network infrastructures by using SDH, Frame Relay (FR) and ATM technologies in order to enter the telecommunication business. Such infrastructures are owned, beside the incumbent operators, by new actors like energy suppliers, railway organisations, city communication operators etc. Now it is important to recognise that such an infrastructure is the basis for entering the business market segment only, since there is no appropriate access network available in order to address the mass market, i.e. the small and medium sized enterprises (SMEs) and the households to offer new multimedia telecommunication services.

Following the ITU-T terminology, an access network is comprising those network entities which provide the required capabilities for the provision of telecommunication services between the local exchange (Lex) and the subscribers; i.e. the related User Network Interface (UNI)'.

\footnotetext{
${ }^{1}$ According to ITU-T recommendation G.902 the access network is allocated between the so-called Service Node Interface (SNI) at the V reference point (at the Lex) and the User-Network Interface (UNI) at the T reference point at the subscriber premises (G.902, 1995). The VB5.1 interface provides access for multiple users of an access network to the service node. The VB5.2 interface has the
} 
Especially the liberalisation of the European telecommunication market January $1^{\text {st }}, 1998$ has resulted in a much stronger competition between network operators as well as manufacturers. Thus, also the access network will be influenced by this new framework. New access network technologies have to be able to offer traditional services very cheap and to realise new multimedia services at the same time with a reasonable price. The most important factors for the design of new technologies for the access network are:

- Minimising the Operation and Maintenance (OAM) costs.

- Improvement of the Quality of Services (QoS) by improving the fault and service management capabilities for example.

- Offering of new services: Incumbent network operators are looking for new revenues and are trying to protect their existing customer base. New operators have the intention to gain market shares by offering new services. Thus, the new access network has to support existing narrowband services like Telephony, ISDN and $64 \mathrm{kbit} / \mathrm{s}$ to $2 \mathrm{Mbit} / \mathrm{s}$ leased line services, as well as new broadband services like fast Internet access and digital video transmission.

Thus, new access networks have to be flexible in order to support the increasing bandwidth demand as well as any type of service.

\section{TODAY'S PSTN-ACCESS NETWORK}

The physical access infrastructure of the classical Public Switched Telephone Network (PSTN) is based on copper twisted pair lines ( $/ \mathrm{b} / \mathrm{b}-\mathrm{lines})$, which connect the subscribers to the next local exchange. In most industrial countries around $99 \%$ of the households are connected by copper twisted pairs to the PSTN in order to offer the classical narrowband telecommunication services. Only in rural areas, wireless access systems are installed as well. Today wireless access networks are investigated in general to offer mobility as an additional service value to the subscribers.

From a main distribution trunk within the local exchange (Lex), cables with some 400 to 600 copper wires are used to finally connect the subscribers with single twisted pairs in a point-to-point star architecture. A typical distance from the Lex to the subscriber is around $4 \mathrm{~km}$. In rural areas some longer distances are used of course.

The PSTN was designed to provide telephony services to the subscribers. The early users needed only the set-up of connections to different locations, so they could talk to each other. This is the Plain Old Telephone Service (POTS). Basically, the provision of POTS is still the rationale behind the telephone network and operators still generate most of their incomes from POTS services.

additional capability of controlling the access network from the service node via a dedicated protocol in such a way that it is possible to have concentration within the access network on a per call basis. 
A digital service, offered today over the PSTN, is realised by the Integrated Services Digital Network (ISDN). ISDN is offering a common interface for voice and data services up to $2 \mathrm{Mbit} / \mathrm{s}$.

Further on, the PSTN today is also used for different data services, like the access to the Internet, by using voice-band modems with capacities of up to 56 $\mathrm{kbit} / \mathrm{s}$ through the $4 \mathrm{kHz}$ voice channel.

As already mentioned above, network operators still generate most of their income from POTS services. However, the need for new more sophisticated services based on Internet traffic will change the importance of the role of the PSTN. This new situation will have a tremendous impact on the architecture and technology of the new access network architecture.

\section{USE OF FIBRE IN THE ACCESS NETWORK}

Optical fibre in the access network is a prerequisite to enable the necessary bandwidth for a final full service network. However, the key question is: how far in the access network is it economically viable to go.

Dependent on the use of fibre in the access network, and thus the location of the optical network termination, i.e. the location of the so called Optical Network Unit (ONU), we have the following network architectures:

- FTTH (Fibre To The Home): The fibre is terminated at the customer premises.

- FTTB (Fibre To The Building): In this case the ONU is located in the basement of the served building, and may be up to $500 \mathrm{~m}$ from the customer apartment or office.

- FTTC (Fibre To The Curb): In this case the ONU may be up to $500 \mathrm{~m}$ from the customer premises. A longer distance between the customer and the ONU requires a much less fibre deployment. Such architectures are also called Fibre to the Cabinet (FTTCa), respectively Fibre to the Node (FTTN), Fibre To The service area (FTTSA) and Fibre to the last amplifier (FTTLA) in CATV networks.

The trend is to bring fibre nearer and nearer to the home in order to increase the capacity, improve the reliability as well as get very low error rates of the network infrastructure. However, FTTH have been proved very expensive, due to the high cost of civil works and the low customer share of optics and electronics equipment.

The new installation of cables, independent whether fibre or copper, is - if no appropriate duct is available - the largest cost factor in a network. FTTC/FFTB offers a good compromise, taking advantage of the high bandwidth of fibre, while utilising the existing asset of the local loop in the last kilometre. In FTTB/FTTC network architectures copper twisted pair or coaxial cable is used to cover the last section to the terminal equipment. The different technological solutions are discussed below.

Optical networks are supporting the transmission of analog as well as digital signals. The network topology of an optical network is either based on a point-topoint configuration or a point-to-multipoint configuration which might result in a 
tree-structure. Based on the use of electrical amplification of the signal within the network we have to differentiate between an Active Optical Network (AON) and a Passive Optical Network (PON).

An optical network (AON or PON) has to be considered as the most promising approach to achieving large-scale full service access network deployment that could meet the evolving service needs of network users. A PON is able to cover some $20 \mathrm{~km}$. This distance depends on the number of used splitting points. A passive optical splitter is generating some power loss which results in a limited distance. Within an AON the optical signal will be amplified within the network which results in a much longer distance. In addition the AON offers additional concentration functions due to the switching capabilities at the splitting points.

\section{ACCESS TECHNOLOGIES FOR MULTIMEDIA SERVICES}

\subsection{The technology choices}

Depending on the physical media used for the access network, different technologies will be employed (ETSI, 1995, Griffith, 1996):

- Twisted-pair copper lines using Digital Subscriber Line (xDSL) technologies like HDSL, ADSL, and VDSL;

- Coaxial cable by using HFC (Hybrid Fibre Coax) technology, where especially the use of fibre in the access network plays an essential role;

- FTTB/FTTC network architectures using copper twisted pair or coaxial cable to cover the last section to the terminal equipment. A combination of fibre/copper is usually called a FITL (Fibre In The Loop) system. A FITL infrastructures is mainly used to offer narrowband services.

Will the fibre be deployed up to the subscriber, a Fibre To The Home (FTTH) access is realised.

- B-ISDN fibre access lines ${ }^{2}$. Since in such a scenario the fibre is always going up to the subscriber, we get a FTTB or a FTTH architecture. On such access lines, usually Metropolitan Area Network (MAN), Frame-Relay or ATM networks are connected.

- Wireless systems, terrestrial or by satellite.

\subsection{Green field situation or use of the existing access infrastructure}

By the development of new access technologies, it is important to note that there are practical and sensible compromises between cost, functions and performance. The choice of the appropriate access technology is based on two factors:

- Services to be offered: narrowband or broadband services, services for households and SMEs or services for large business users.

\footnotetext{
${ }^{2}$ B-ISDN UNI specifications are available for $155 \mathrm{Mbit} / \mathrm{s}, 622 \mathrm{Mbit} / \mathrm{s}, 1.5 \mathrm{Mbit} / \mathrm{s}, 2 \mathrm{Mbit} / \mathrm{s}, 51 \mathrm{Mbit} / \mathrm{s}$, and 25.6 Mbit/s; see ITU-T recommendation I.432.1-5.
} 
- Deployment of a new access infrastructure or reuse of an existing access infrastructure. In a so called ,green field situation" there is no existing access infrastructure available. However, such a situation is not very often the case in industrial countries.

In a green field situation FITL- or HFC-networks are deployed usually. HFC technology will be used if video broadcast is part of the offered service. A FITL access infrastructure is appropriate if narrowband services like POTS, ISDN and leased line services up to $2 \mathrm{Mbit} / \mathrm{s}$ are of main importance. If a quick service offering of narrowband services and mobility is of main importance, wireless technologies are appropriate.

The owners of access infrastructures (copper or coax) are driven to capture profits from new services. In this way, they might try to invest as little as possible and go for immediate results. In this approach, the linearity of the investment against the possible return is important. This is even more true looking to the uncertainty of market share for new services. Thus the reusability of the already available access infrastructure is of main importance. For the reuse of an existing physical infrastructure (coax cable or copper twisted pair), the following technologies have to be considered:

- HFC-technology, if video broadcast services are part of the offered services.

- FITL-technology if some part of the existing copper infrastructure has to be upgraded by fibre and the offered services are mainly POTS, ISDN and nx64 $\mathrm{kbit} / \mathrm{s}$ (up to $2 \mathrm{Mbit} / \mathrm{s}$ ) leased line services.

- xDSL-technologies, if broadband services to the subscriber have to be offered via the point-to-point twisted-pair copper lines.

The different technological solutions are discussed in the following.

\subsection{Direct fibre access (FFTH/FTTB) and Fibre In The Loop (FITL) systems}

As highlighted above, a direct broadband fibre access (FTTH/FTTB) which offers an enormous capacity for the offering of multimedia services, is economically just feasible for very large business customers, which are requesting symmetrical access and high capacity to a backbone network. However, recent standardisation activities are aiming at developing very cost effective optical network terminations (FSAN, 1997). Such a cheap optical network termination is the prerequisite for realising any cost effective FFTH infrastructure in the future.

A FITL (Fibre In The Loop) system is a combination of fibre cables feeding neighbourhood Optical Network Units (ONUs) and last mile connections by existing or new copper wires. A FITL infrastructure is mainly used to offer narrowband services like POTS, ISDN and leased line services up to $2 \mathrm{Mbit} / \mathrm{s}$. The Deutsche Bundespost Telekom project in the eastern part of Germany is a good example of a large FITL deployment, based on a PON architecture, in a green field situation (Hytas, 1995). 
The access technologies which are based on the reuse of existing infrastructure and which are the basis for offering multimedia services on a broad range to the subscribers are discussed in the following sections: xDSL and HFC.

\section{DIGITAL SUBSCRIBER LINE (DSL) TECHNOLOGIES}

\section{1 xDSL Technologies: HDSL, ADSL, VDSL}

It is important to note that ISDN was the first technology to transport digital signals up to $2 \mathrm{Mbit} / \mathrm{s}$ over the twisted pair access. The new available Digital Subscriber Line (DSL) transmission techniques like HDSL (High bit rate Digital Subscriber Line), ADSL (Asymmetric Digital Subscriber Line) and VDSL (Very high speed Digital Subscriber Line) can deliver data at multi-Mbit/s over the unscreened, twisted telephone wires, originally intended for bandwidths of between $300 \mathrm{~Hz}$ and $3,4 \mathrm{kHz}$. This is due the remarkable advances in digital signal processing technology. These allow the implementation of sophisticated channel modulation techniques which suggest that there are no fundamental technological barriers to overcome, at least on the digital side. In fact the challenges are mainly of an analog nature, set by the external electrical environments; i.e. the imposed noise interference which will degrade the Signal-to-Noise Ratio (SNR).

For the development of these new technologies, emphasis is not only given to maximum bitrate possibilities, but also on the robustness of the physical transport medium as well as different service chiaracteristics. For that reason, different modulation techniques are used throughout the solutions.

\section{HDSL (High bit rate Digital Subscriber Line)}

HDSL realises the transmission of $2 \mathrm{Mbit} / \mathrm{s}$ over a copper twisted pair in both directions (upstream and downstream) and offers usually a standardised G.703 interface to the subscriber. To improve the max. possible distance for the transmission of the $2 \mathrm{Mbit} / \mathrm{s}, 2$ or 3 copper twisted pairs can be used in parallel. Since the bandwidth capacity is the same in both directions, this is also called a SDSL (Symmetric Digital Subscriber Line) technology. HDSL is becoming a well accepted technology to offer especially for business customers $2 \mathrm{Mbit} / \mathrm{s}$ leased line services $^{3}$. The use of the HDSL technology for the realisation of a multimedia application is described in (Leopold, 1996).

\section{ADSL (Asymmetric Digital Subscriber Line)}

An ADSL system connects an ADSL modem pair through a twisted pair copper line, creating a high bit rate downstream channel and a medium bit rate upstream channel (Chen, 1994). The high bit rate downstream channel ranges from 1,5 - 7,5 Mbit/s, while the upstream channel ranges from 16 to $640 \mathrm{kbit} / \mathrm{s}$. This is achieved without disturbing the POTS service already installed on the line. The POTS

\footnotetext{
${ }^{3}$ Also $64 \mathrm{kbit} / \mathrm{s}$ leased line services are technically feasible; however there is no clear market requirement for such a service up to now.
} 
compatibility is achieved by using a higher frequency band for the digital signals than used for the analog telephone signal (i.e. above $4 \mathrm{kHz}$ up to $1 \mathrm{MHz}$ ). The analog signals are separated from the digital signals by a so called „POTS splitter“.

The downstream high-speed channel is based on the assumption that most residential high-speed services will be asymmetric. The business users requiring symmetric services will install fibre for bi-directional data transfer. The downstream data rates depend on a number of factors, including the length of the copper line, its wire gauge and cross-coupled interference. Line attenuation increases with line length and frequency and decreases as wire diameter increases.

\section{VDSL (Very high speed Digital Subscriber Line)}

VDSL is a complimentary development of the ADSL technology. By use of VDSL a much higher bandwidth will be achieved by a much less distance to the customer: up to $25 \mathrm{Mbit} / \mathrm{s}$ in downstream direction and up to $2 \mathrm{Mbit} / \mathrm{s}$ upstream direction at distances of up to $1 \mathrm{~km}$. This dimensioning makes the VDSL technology to a good extension of a FTTCa-architecture. However, VDSL is still in the definition phase and further developments have to be expected.

\section{ISDL (ISDN Digital Subscriber Line)}

A further xDSL variant is to integrate ISDN and ADSL. However, such an ISDN Digital Subscriber Line (ISDL) approach is only useful for the integration with an ISDN basic access. The ISDN-primary access ( $2 \mathrm{Mbit} / \mathrm{s}$ ) is usually used to connect PABXs with the PSTN. The ADSL technology has a complete different objective: to bring multimedia capacity to the subscriber (i.e. up to $6 \mathrm{Mbit} / \mathrm{s}$ ). However, standards are not finalised yet, and the market acceptance of such an integration has to be verified very carefully.

\section{ADSL-Lite}

In order to improve the market acceptance, a special ADSL variant has been developed. ADSL-Lite has the objective to reduce the installation effort at the subscriber premises by allowing a simple plug in by the subscriber in any walloutlet in the home, just as usual base-band modems. This is achieved by eliminating the POTS splitter, but results also in a compromise concerning the ADSL performance.

\subsection{Modulation Techniques}

In fact modern coding and modulation techniques provide a level of performance that approaches the theoretical limit of the physical bandwidth. New ADSL developments are using Discrete Multi-Tone (DMT) modulation techniques. DMT is a form of multicarrier modulation. In the frequency domain, DMT divides the channel into a large number of sub-channels. Only those frequency channels will be used which are not disturbed. These channels will be identified during the initialisation phase and will be permanently checked again during operation in order to adapt to a changing environment (i.e. highly robust in noisy environments). This technique is called rate adaptive ADSL (RADSL). 
Another technique, the so-called Carrier Amplitude Phase (CAP) modulation technique, is under discussion within standardisation as well. However, DMT is an accepted technology and thus standardised within ANSI T1E1.4.

\subsection{ADSL and ATM Integration}

Modern ADSL products, are integrating ATM and ADSL technology. The main advantages by such an architecture in the access network are the following:

- Support of different traffic characteristics (continuous bit streams, data bursts, etc.);

- Any granularity of bit rate for different user channels.

- Multiple QoS levels per subscriber (ATM connections), allowing a service range from low cost residential to premium business at a guaranteed quality level.

- Combining DMT and ATM provides a flexible bit rate, exploiting the maximum line transfer capacity.

- The offering of different interface types. Beside Ethernet for the traditional connectivity of PCs and Network Computers (NCs), the ATM-Forum 25,6 $\mathrm{Mbit} / \mathrm{s}$ has been established as a new broadband standard interface at the users premises.

\subsection{ADSL System Architecture}

The ADSL access network encompasses the ADSL modems and the access multiplexer system at the local exchange (Lex) and the ADSL modems at customer premise connected via the local loop. The ADSL modem at the Lex side is also called the ADSL-LT (line termination) and the modem at the subscriber premises is called ADSL-NT (network termination).

The access multiplexer system and the modems at the Lex side are usually combined into a single unit called the access node (using the ADSL Forum terminology) and also referred to as the "DSLAM" (DSL Access Multiplexer) or Subscriber Access Multiplexer (SAM). When the backbone network is ATM, the access node is connected to an ATM access switch. The ADSL access node and ATM access switch may or may not be co-located. The function of the ATM access switch is to concentrate and switch traffic from a number of access nodes onto the regional broadband network. The ADSL access node (DSLAM) performs the following functions:

- Line Termination (LT) of the ADSL subscriber lines.

- Concentration/multiplexing of the ADSL subscriber lines towards the broadband network. WAN interfaces such as a STM-1 are expensive resources for an operator. It is important to concentrate as many subscriber lines as possible onto a single network interface. A multiplexing scheme that provides high concentration while guaranteeing the individually negotiated QoS will be an important asset for network operators, because it will allow them to offer differentiated services at a reasonable cost. 
- Termination of customer ATM signalling channels. To provide a standard, scaleable mechanism for supporting switched virtual circuit service to ADSL customers, the ADSL access node should terminate the ATM signalling protocol from each ADSL customer, and generate a single standard UNI signalling interface toward the access ATM switch.

At the subscriber premises the installation of a network termination (ADSL-NT) is required to which a LAN, a PC, a Network Computer (NC) or a TV set with an appropriate Set Top Box (STB) is connected.

As shown in Figure 3, the subscriber has now the usual PSTN access for POTS services, an access to the Internet and On-line services to an Internet Service Provider (ISP) as well as an access to digital video contents.

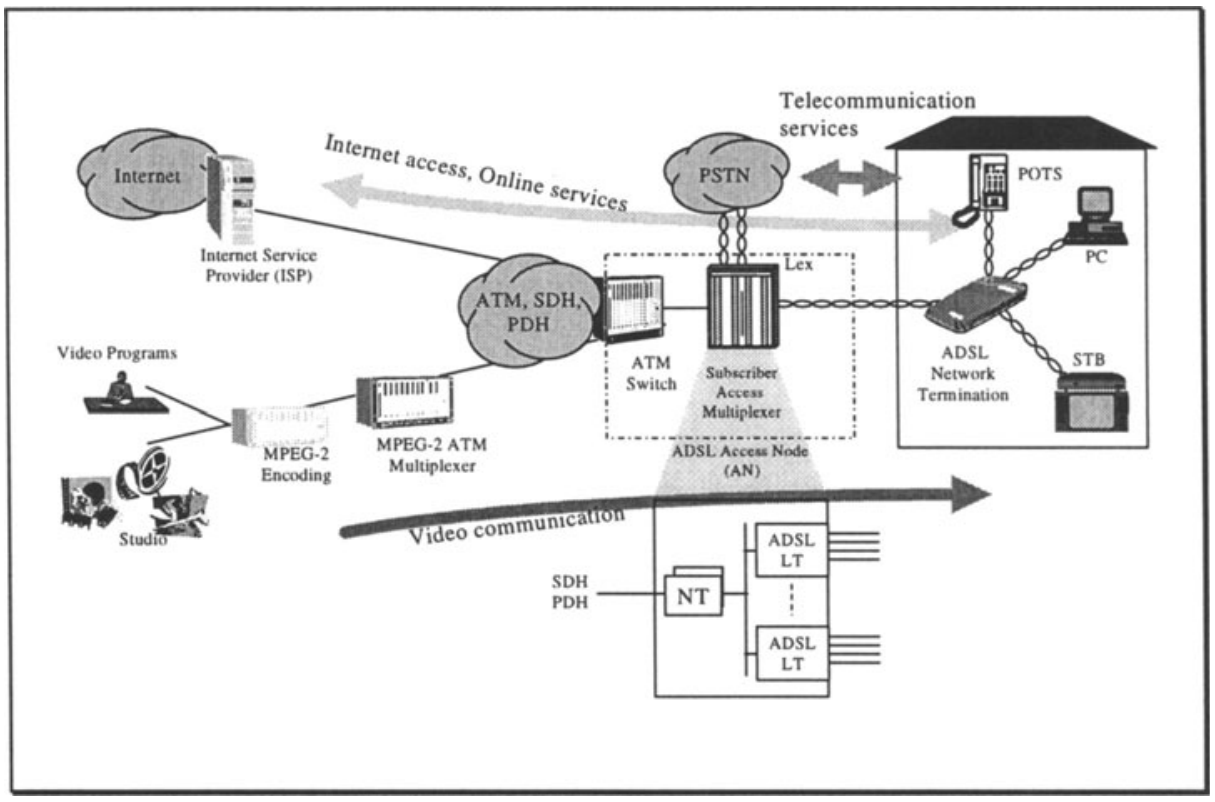

Figure 1. ADSL network architecture

\subsection{The business opportunities of xDSL Technologies}

To summarise, the business opportunities of xDSL-Technologies are:

- Use of the existing PSTN access network resulting in low installation cost which allows the quick connection of new users and allows early service deployment.

- Costs are strictly proportional with the number of connected customers. Thus, a low upfront investment is necessary at the one hand, which limits the financial risk of the operator. 
- Sufficient capacity for multimedia services with no impact on the telephony service at the same line.

- Bandwidth asymmetry is well suited for typical client/server applications.

\section{CATV-OPERATORS AND HYBRID FIBRE COAX (HFC) ACCESS NETWORKS}

\subsection{The CATV business}

Basically the broadcast TV business is „entertainment“. Thus, the customer is not really willing to pay for the transmission of signals, but he is prepared to pay for the content he receives. Comparing to the traditional telecom business, other revenues than transmission tariffs are necessary. Thus the prime revenue streams are based on advertising, licenses, basic cable fees, and pay TV. However, it has to be noted, that the traditional TV business is a low margin business with long return of investments.

It seems to be obvious that CATV operators have to protect first of all their core business against Direct Broadcast Satellite (DBS) services especially stimulated by the new digital TV services, while exploring new business areas like data communications, Internet access, online services, and to look for new market shares in the classical telecommunication business to ensure growing revenues in the long term.

However, the main problem that CATV operators have to consider is the move in mindset needed to take advantage of the new market opportunities. Entering the new telecommunication business means more than just a technological upgrading of the network infrastructure. It also means the definition of new services and further on establishing relationships with customers through marketing, customer service, etc. CATV operators have to learn to think more like telecommunications companies. Such a need for cultural change is a potential critical factor for CATV operators.

\subsection{The new business opportunity of a HFC network}

Traditional CATV networks make extensive use of coaxial cooper cable to carry the signal from the head-end (HE) to the subscriber. Along the path amplifiers are deployed to compensate for the attenuation of the long coax cable lines. Each broadcast TV signal takes $6 \mathrm{MHz}$ for a NTSC signal or $8 \mathrm{MHz}$ for a PAL signal here in Europe. The bandwidth available on these pure coax networks usually range from 300 to $500 \mathrm{MHz}$.

During the 80s and 90s cable operators began upgrading their networks to a Hybrid Fibre Coax (HFC) architecture to provide higher quality, increased programming, and new services. These new networks combine high capacity fibre lines with inexpensive coax lines and are the basis for establishing bi-directional capabilities. 
HFC based networks have Broadband Optical Network Terminations (BONTs) that typically serve some hundred to some thousand homes by a coax cable; i.e. serving area of a so called "coax cell". 500 homes per coax cell are seen as the optimised configuration to offer the whole set of possible services from analogue broadcast TV to professional telecommunication services. Such a modern HFC network is able to offer the following set of services for example:

- 40 analogue TV broadcast channels,

- 200 broadcast digital TV channels,

- 400 on-demand channels (Near Video On Demand (NVOD, etc.)),

- Internet access via the TV set,

- Internet access and high-speed digital data services via LANs/PCs, and voice telephony services.

Moving to full service providers, digital TV, data communications, Internet access, on-line services, and telephony services, allow CATV operators to grow their business by

- protecting their core business against the DBS competition,

- entering new business areas,

- competing with the traditional telecom operators, and

- offering the access for the „last mile“ for other network operators which don't have direct access to the customers.

\subsection{Services to be offered on CATV networks}

There are four service classes to be offered on CATV networks. Figure 2 shows the different functional blocks at the subscriber premises:

\section{Analogue TV broadcast}

This is the traditional means of transmitting analogue TV signals to subscribers. Analogue audio distribution and information services via Teletext are established as a well accepted services by the subscribers. The Teletext service is based on the distribution of information (text and graphics) in a broadcasting communication mode without any user interactivity over the network.

Based on these broadcast services, either analogue or digital, first interactivity is realised basically by using the PSTN POTS service. Thus new services like Home Order Television (HOT) which allows the so called "impulse tele-shopping" applications, and information on demand services based on Teletext are offered.

Pay-TV services based on a simple scrambling of the analogue TV signals, is one of the new services being offered by many CATV operators. For transportation of the control information form the subscriber to the CATV operator, usually the POTS service of the PSTN is used. For such a Pay-TV service, the customer requires an analogue STB in order to decode the scrambled TV signal.

\footnotetext{
${ }^{4}$ The functional modules do not necessarily relate to implementations; i.e. several functional modules might be combined within one implementation in the future.

${ }^{5}$ E.g. The Pay-TV channel Premiere.
} 


\section{Digital TV and interactive multimedia services}

For digital TV broadcast based on the Digital Video Broadcast (DVB) standards and for interactive multimedia services based on the Digital Audio Video Council (DAVIC) specifications, the subscriber requires a DVB compliant digital STB in order to decode the digital MPEG-2 encoded video streams. Digital TV broadcast (audio and video) is basically offering the same services as for the analogue signal transmission. However, due to the digital transmission a very much higher capacity in the network is achieved. Dependent on the coding schema and the used bandwidth for the digital video signal ${ }^{6}$, this allows the transmission of approximately 8 digital channels per $8 \mathrm{MHz}^{7}$. Thus Near Video on Demand (NVOD), and distribution of selected programs to a specific set of customers are the major services planned. For such services, no interactivity over the network is required.

If the CATV network is offering bi-directional functionality, the communication from the customer to the CATV operator is done via the CATV network, otherwise the use of the PSTN is possible as well.

Based on such interactive services, Pay per View (PPV), Internet access, on-line services and interactive multimedia services can be offered to the subscriber (Furth, 1995).

\section{Cable telephony services}

Real interactivity based on a return channel within the CATV infrastructure is necessary for the offering of telecommunication services like POTS, ISDN and 64 $\mathrm{kbit} / \mathrm{s}$ to $2 \mathrm{Mbit} / \mathrm{s}$ leased line services. These services can be seen as „classical telecom services", offering guaranteed QoS, which are implemented over the CATV infrastructure by a so called „cable telephony“ system (Fletcher, 1997). All services are based on $64 \mathrm{kbit} / \mathrm{s}$ channels. At the subscriber premises a so called Coax Network Termination (CNT) is installed to offer the different interfaces for POTS, ISDN and the nx64 kbit/s leased line services. The bandwidth capacity is usually below $2 \mathrm{Mbit} / \mathrm{s}$ in both directions. This service class allows the CATV operator to get into the lucrative telephony business.

\section{Data communication services}

These service class is based on offering LAN interfaces at the subscriber premises like Ethernet in order to allow Internet access and online-services by the use of a PC and LAN interconnection to realise intranetworks. This is implemented by a so called ,cable data modem" system.

It is important to note, that the users of a cable data modem systems are using a shared medium (i.e. a legacy LAN), which means, that the offered bandwidth of the $10 \mathrm{Mbit} / \mathrm{s}$ Ethernet for example, have to be shared by all users related to this LAN (Laubach, 1996). Like in a legacy LAN environment, there are no QoS guarantees provided by the network.

\footnotetext{
${ }^{6}$ The transmission bandwidth required (e.g. 2, 4, 6 or $8 \mathrm{Mbit} / \mathrm{s}$ ), depends on the intended video quality.

${ }^{7}$ By the use of a QAM-64 modulation, $32 \mathrm{Mbit} / \mathrm{s}$ can be transported within a $8 \mathrm{MHz}$ channel.
} 
During the last few years much effort has been made by international organisations and companies to build suitable physical and access protocols (MAC) for interactive services using the CATV network for data communications and the development is still ongoing. Within the European Digital Video Broadcasting (DVB) Project the Return-Channel Working Group has developed a complete specification for a physical and MAC protocol in cooperation with the Digital Audio Council (DAVIC).

In North America several large cable companies have joined forces to build the Multimedia Cable Network Systems (MCNS). MCNS have set up the Data Over Cable Service Interface Specification (DOCSIS). IEEE has created a working group 802.14 that is also developing a standard for a physical and a MAC protocol. The MCNS is focusing on the pure Internet environment and is advanced very well, while the DVB/DAVIC and the IEEE activities are focusing on the use of ATM. Which standard will succeed on the market finally is still not clear.

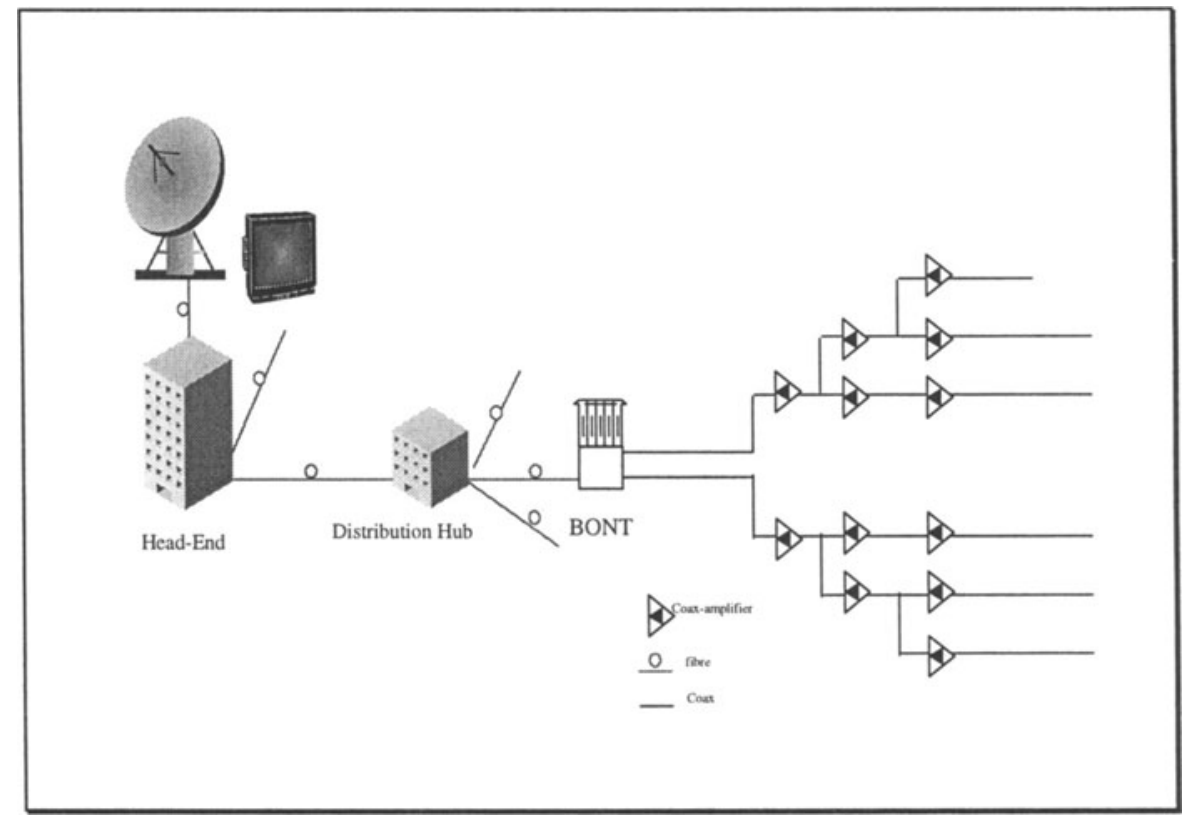

Figure 2: Services over the CATV infrastructure

\section{ATM on HFC Networks}

In order to support the different traffic characteristics by a flexible communication architecture, the use of ATM in HFC networks is investigated within the EU ACTS project ATHOC. For more details refer to Böttle, 1997a and Böttle, 1997b. 


\subsection{Evolution of CATV to modern Telecommunication Networks: HFC}

To allow the realisation of new services, CATV network infrastructures have to be upgraded in order to provide new capabilities for the subscribers. The starting point for the CATV operators are the existing coax architecture. Purely coaxial CATV networks evolve to Hybrid Fibre Coax (HFC) networks, by implementing

- a bi-directional network, with capacity for interactive services,

- new electrical amplifiers for the coax cable infrastructure, working up to 800 $\mathrm{MHz}$ to offer enough capacity for all services, and

- a fibre overlay network to improve the reliability and to bring the transmission capacity as near as possible to the customer.

\section{HFC - Physical Network Infrastructure}

The Hybrid Fibre Coaxial (HFC) architecture has become a standard for CATV operators. This architecture does not contain switching elements in the distribution network, and only requires optical-to-electrical conversion, amplification and power splitting. Thus every customer receives the same signal, which contains the information of all the services provided.

A typical HFC network has a fibre star point-to-multipoint subnetwork and a tree-and-branch coaxial subnetwork. The Head-End (HE) receives, modulates and transmits the CATV channels over the fibre network towards the Broadband Optical Network Termination (BONT), where the signals are converted back to electrical signals to serve the customers by coaxial cable. Thus, from a physical point of view, a HFC network is made of two parts:

- An optical network, which runs from the optical transmitter at the network head-end to a point close to the customer, in a star or multiple star architecture. The fibre part of the HFC network is a Passive Optical Network (PON) or an Active optical network (AON) usually with two fibres, one for each direction of transmission (also called „two fibre system“). Using optical fibres to a large extend, instead of the coax infrastructure is improving in addition reliability and maintenance expenses.

- A coaxial branch-and-tree network, which connect the termination of the optical network (BONT) with each customer. The BONT performs the transition between multiple main coax cable and the optical fibre. If the serving area of the coax infrastructure is up to $250 \mathrm{~m}$ only, no amplifiers are used. This is the recommended approach for green field situations.

Typically some intermediate points between the head-end and the BONTs have been introduced which allow a hierarchical structuring of the fibre distribution network; so called Distribution Hubs (DH) (see Figure 3). These nodes perform optical signal amplification and optical signal splitting. These nodes could be seen as equivalent to the local exchanges of the telephone networks and might be the points where new equipment for the transmission of new services will be placed 
(further obvious locations for the new equipment are the $\mathrm{HE}$ as well as the $\mathrm{BONT}^{8}$ ).

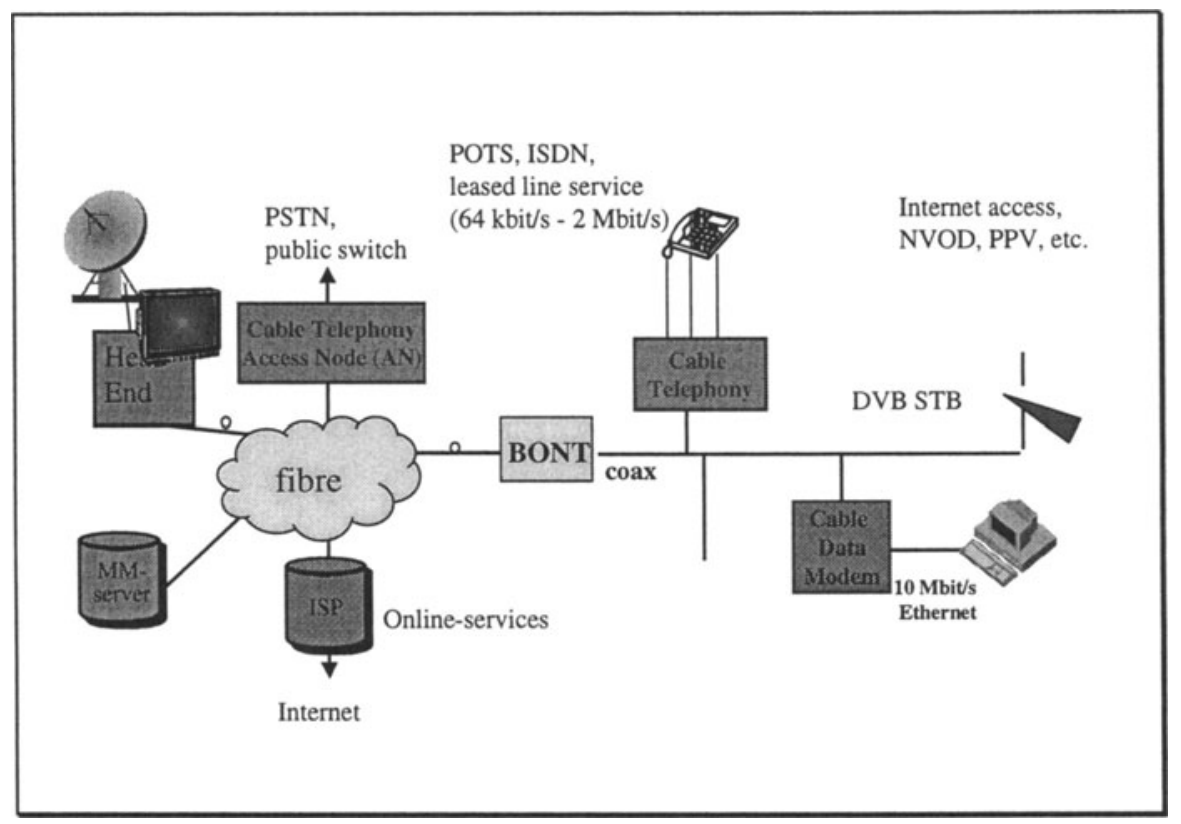

Figure 3. HFC network architecture: HE, DH, BONTs, coax amplifiers (LE)

\section{Signal transmission}

A HFC network transports the information (analogue and digital signals) by using frequency multiplexed analogue carriers (FDM). Each TV channel is modulated on a different Radio Frequency (RF) carrier; allocating $8 \mathrm{MHz}$ per analogue TV channel (European standard; PAL).

In a traditional CATV network, usually the transmission is just done unidirectional from the network to the user (downstream) by a broadcast communication mode (since the very first usage of this infrastructure is analogue video distribution). This limitation is usually due to the uni-directional amplifiers within the coax network. These amplifiers are working in the frequency range up to $302,446,600$ or $860 \mathrm{MHz}$. After upgrading or replacing all the uni-directional coax-amplifiers, the infrastructure is able to transport the signals bi-directional. However, the signals transmission is not symmetrical.

The upper part of the spectrum is used for downstream transmission and the signal is distributed to all subscribers in a broadcast communication mode. Encryption and/or other measures to control the access to the information have to be used for services other then broadcast TV to ensure appropriate security. The

\footnotetext{
${ }^{8}$ Although usually there is no space for additional equipment available at the BONT location.
} 
lower part of the spectrum is used for upstream transmission. A dedicated protocol is required for sharing the available capacity (see below).

\section{Upstream signal transmission (return channel)}

The return channel employed will have significant impact on the services offered and their degree of interactivity. Although it is not imperative to implement the return channel in the same network as the downstream channels, doing so leads to a homogeneous and user friendly solution.

The upstream signals that must be transmitted are: network supervision signals, alarms, etc. coming from coax amplifiers, BONTs, DHs, or other network elements dependent on the service implemented on the HFC network, in order to integrate the HFC part in a powerful network management system, and signals for the interactive services like voice channels for telephony services, upstream channels for data services, return channel for interactive multimedia services.

In the CATV network the physical medium - the coaxial cable - is shared by all subscribers. Therefore, an access procedure is required which provides collision free access to the shared medium and assigns capacity according to the customer's requirements. Time division multiple access (TDMA) and frequency division multiplex (FDM) techniques are used to allow the return path to be shared among the subscribers.

The return channel of the CATV network has some impairments which has to be considered for the final system design:

- Limited bandwidth (some $50 \mathrm{MHz}$ only) and modulation with low efficiency due to the usage of a robust modulation technique (a typical spectral efficiency of $1-2$ bits/Herz.

- Noise accumulation in the upstream direction from each customer termination with a coax cell. Common sources for external interference are radio signals coupled to the network, engine based interference, TV receivers, etc.

\section{Downstream signal transmission}

The downstream channel has a distribution architecture. No noise accumulation from different terminations is performed. For analogue signal transmission, the same modulation technique for terrestrial TV and FM radio services is used for compatibility reasons. For digital services (telephony, data communications, digital TV), a modulation technique providing high spectrum efficiency can be used. Typical examples are 64-, 32-, 64-, 128-, 256-QAM. These modulation techniques provide a spectrum efficiency up to 6 bits/Herz, thus resulting in several Mbps on a $8 \mathrm{MHz}$ channel'.

According to the current standardisation discussions, $41 \mathrm{Mbit} / \mathrm{s}$ can be transmitted by means of 64-QAM in an $8 \mathrm{MHz}$ wide CATV channel. Considering the redundancy needed for error protection (by a 204,188) Reed-Solomon code, 38 $\mathrm{Mbit} / \mathrm{s}$ remain for the transmission of user data.

\footnotetext{
${ }^{9}$ Capacity in a $8 \mathrm{MHz}$ channel: 16-QAM: $25 \mathrm{Mbps}$; 32-QAM: $32 \mathrm{Mbps}$; 64-QAM: $38 \mathrm{Mbps}$; 128QAM: $45 \mathrm{Mbps}$; although it has to be noted that these figures various from the different product implementations.
} 


\section{CONCLUSION}

Based on the studies conducted by the FSAN (FSAN, 1997), it was determined that the per-line cost of producing a full service access network will slowly decrease with volume of production. However, at a sufficiently high volume level the development of new technologies becomes justified that can enable significant reductions in per-line equipment and installation costs.

However, no one knows for certain what path is socially optimal for residential adoption of broadband technology. Whatever the predictions, business decisions must be made in both the public and private sectors to determine the next step. Telcos and CATV operators have to continue to upgrade their networks both to achieve network efficiency and to compete in the near term for new and advanced communication services (Potts, 1997). Especially the new operators have to consider:

- The installation of a wireless access system suffers from the limited capacity not supporting multimedia applications currently.

- A new installation of an access infrastructure might be too expensive and thus economically not feasible.

- The use of the copper lines of the PSTN access infrastructure from the incumbent operator ("unbundling") depends on the interconnection fees.

- The incumbent operators have a powerful potential access infrastructures by the use of XDSL technologies.

- The use of the available CATV infrastructure might be an economical solution.

The access network has a very essential role within a telecommunication infrastructure, and thus it brings an added value which is much more than just the transmission of information between the subscriber and the backbone network. Next steps should aim at demonstrating the availability of technologies and products for broadband access networks realising full service capabilities. This should also contribute to the effort in standardisation to enhance the definition for further system developments.

Finally, it is important to note that only at a sufficiently high volume level the development of new technologies becomes justified. Thus a powerful access network infrastructure, which supports multimedia group communication services to a broad range of users; i.e. households, SMEs and business units, will be the prerequisite for any mass deployment of future multimedia services. 


\section{REFERENCES}

Berkowitz, P. (1997) The Changing Shape of the Access Network. TELECOMMUNICATIONS, Vol. 31, No. 10, October 1997 (pp 109-114).

Böttle D. (1997a) Network Architecture for an ATM based Hybrid Fibre Coax System and related Applications. CWAS'97 International Workshop on Copper Wire Access Systems, Budapest, Hungary, October 27-29, 1997.

Böttle, D. and Wahl, S. and Sierens, Chr. And Bastos J. and Borges I. and Frei P. and Christ, P. and Fahner H. and Ramlot G. (1997b) ATM Applications over Hybrid Fibre Coax Trials. ISS'97, Toronto, September 21-26, 1997.

Chen, W.Y. and Waring, D.L. (1994) Applicability of ADSL to Support Video Dial Tone in the Copper Loop. IEEE Communications Magazine, May 1994 (pp.102-109).

ETSI (1995) Video on Demand Network Aspects. ETSI/NA5, DTR/NA-52109, Technical Report, October 1995.

Fletcher, M. (1997) Cable Telephony: Coming to Market. TELECOMMUNICATIONS, Vol. 31, No. 9, September 1997 (pp 81-83).

FSAN (1997) Full Service Access Network (FSAN) Gx Initiative, summary of results, 25 February, 1997.

Furth, B. and Kalra, D. and Kitson F.L. and Rodriguez, A.A. and Wall, W.E. (1995) Design Issues for Interactive Television Systems. COMPUTER, May 1995.

G.902 (1995) Access Networks - Architectures, Services, Arrangement and Service Node Aspects. ITU-T Recommendation, COM 13 -R41, July 1995.

Griffith, M. and Guirao, F. and Van Noorden, L. (1996) Network Evolution for residential Broadband Interactive Services - From RACE to ACTS. European Conference on Multimedia Applications, Services and Techniques (ECMAST 96), Proceedings Part I, May 1996.

HYTAS (1995) HYTAS: Ein zukunftsorientierter Netzzugang für Multimediadienste", Vortrag zur 36. Post- und Fernmeldetechnischen Fachtagung des VDPI - Multimedia - anbieten, transportieren, anwenden", ke Kommunikations-Elektronik GmbH \& Co, Februar 1995.

Laubach, M. (1996) To foster residential area broadband internet technology: IP datagrams keep going, and going, and going .... Computer Communications 19, 1996 (pp. 867-875).

Leopold, H. and Hirn, R. The Bookshop Project: An Interactive Multimedia Application Case Study. In Proc. of the International COST237 Workshop on Multimedia Transport and Teleservices, D. Hutchison, A. Danthine, H. Leopold, G. Coulson (eds), Barcelona, Spain, November 1996 (Springer Verlag LNCS 1185, ISBN 3-540-62096-6).

Potts, M (1997) Guideline NIG-G1: Broadband Deployment. ACTS, Network Integration Chain Group, September 1997 (http://ginaiihe.ac.be/).

Sales, B. and Dumortier, P. and Van Mieghem, P. (1998) Dual-Mode Routing: A long term Strategy for IP over ATM. 6 ${ }^{\text {th }}$ IEE International Conference on Telecommunications, Edinburgh, 29.3-1.4.1998. 


\section{BIOGRAPHY}

Helmut Leopold, born April 27th, 1963, in Hohenems, Austria, made his degree in 1982 in Electronic and Communications at the Technical College in Rankweil, Austria. In 1989 he made the degree of Dipl.-Ing. Informatik (Computer Science) at the University of Technology of Vienna. From 1989 to 1994 he was responsible for the group „Multimedia Communications“ at Alcatel Austria Research Center in Vienna and was actively involved in international standardisation and in European R\&D projects in the broadband communication area. Since 1994, Mr. Leopold is with Alcatel Austria AG, extending his activities on marketing and usage of new multimedia services based on broadband technologies. Since 1996 he is account manager for the CATV-market in Austria. 